\title{
THE RELATION BETWEEN MAXWELL, DIRAC, AND THE SEIBERG-WITTEN EQUATIONS
}

\author{
WALDYR A. RODRIGUES JR.
}

Received 22 October 2002 and in revised form 26 December 2002

\begin{abstract}
We discuss unsuspected relations between Maxwell, Dirac, and the Seiberg-Witten equations. First, we present the Maxwell-Dirac equivalence (MDE) of the first kind. Crucial to that proposed equivalence is the possibility of solving for $\psi$ (a representative on a given spinorial frame of a Dirac-Hestenes spinor field) the equation $F=\psi \gamma_{21} \tilde{\psi}$, where $F$ is a given electromagnetic field. Such task is presented and it permits to clarify some objections to the MDE which claim that no MDE may exist because $F$ has six (real) degrees of freedom and $\psi$ has eight (real) degrees of freedom. Also, we review the generalized Maxwell equation describing charges and monopoles. The enterprise is worth, even if there is no evidence until now for magnetic monopoles, because there are at least two faithful field equations that have the form of the generalized Maxwell equations. One is the generalized Hertz potential field equation (which we discuss in detail) associated with Maxwell theory and the other is a (nonlinear) equation (of the generalized Maxwell type) satisfied by the 2-form field part of a Dirac-Hestenes spinor field that solves the Dirac-Hestenes equation for a free electron. This is a new result which can also be called MDE of the second kind. Finally, we use the MDE of the first kind together with a reasonable hypothesis to give a derivation of the famous Seiberg-Witten equations on Minkowski spacetime. A physical interpretation for those equations is proposed.
\end{abstract}

2000 Mathematics Subject Classification: 81Q05, 81R25, 15 A66.

1. Introduction. In [1, 2, 3, 4, 5], using standard covariant spinor fields, Campolattaro proposed that Maxwell equations are equivalent to a nonlinear Dirac-like equation. The subject has been further developed in [35, 39] using the Clifford bundle formalism, which is discussed together with some of its applications in a series of papers, for example, $[11,12,13,17,18,19,22,26$, $28,29,30,35,39,40]$. The crucial point in proving the Maxwell-Dirac equivalence (MDE) starts once we observe that to any given representative of a DiracHestenes spinor field (for more information, see Section 2, and for details, see $[17,22,26,30]) \psi \in \sec \left[\bigwedge^{0}(M)+\bigwedge^{2}(M)+\Lambda^{4}(M)\right] \subset \sec \mathscr{C} \ell(M, g)$, there is an associated electromagnetic field $F \in \sec \bigwedge^{2}(M) \subset \sec \mathscr{C} \ell(M, g)\left(F^{2} \neq 0\right)$ through the Rainich-Misner theorem [25, 35, 39, 40] by

$$
F=\psi \gamma_{21} \tilde{\psi}
$$


Before proceeding, we recall that for null fields, that is, $F^{2}=0$, the spinor associated with $F$ through (1.1) must be a Majorana spinor field [17, 18, 39], but we do not need such concept in this paper. Now, since an electromagnetic field $F$ satisfying Maxwell equation has six degrees of freedom and a DiracHestenes spinor field has eight (real) degrees of freedom, some authors felt uncomfortable with the approach used in [35, 40], where some gauge conditions have been imposed on a nonlinear equation (equivalent to Maxwell equation), thereby transforming it into usual linear Dirac equation (called the Dirac-Hestenes equation in the Clifford bundle formalism). The claim, for example, in [14] is that the MDE found in [35, 40] cannot be general. The argument is that the imposition of gauge conditions implies that an $\psi$ satisfying (1.1) can have only six (real) degrees of freedom, and this implies that the DiracHestenes equation corresponding to Maxwell equation can be only satisfied by a restricted class of Dirac-Hestenes spinor fields, namely, the ones that have six degrees of freedom.

Incidentally, in [14], it is also claimed that the generalized Maxwell equation

$$
\partial F=J_{e}+\gamma_{5} J_{m}
$$

(where $J_{e}, J_{m} \in \sec \bigwedge^{1}(M)$ ) describing the electromagnetic field generated by charges and monopoles [19] cannot hold in the Clifford bundle formalism because according to that author the formalism implies that $J_{m}=0$.

In what follows, we analyze these claims of [14] and prove that they are wrong (Section 3). The reasons for our enterprise is that, as will become clear in what follows, understanding (1.1) and (1.2) together with some reasonable hypothesis permits a derivation and even a possible physical interpretation of the famous Seiberg-Witten monopole equations [21, 24, 36]. So, our plan is the following. First we introduce in Section 2 the mathematical formalism used in the paper, showing how to write Maxwell and Dirac equations using Clifford fields. We also introduce Weyl spinor fields and parity operators in the Clifford bundle formalism. In Section 3, we prove that, given $F$ in (1.1), we can solve that equation for $\psi$, and we find that $\psi$ has eight degrees of freedom, two of them being undetermined, the indetermination being related to the elements of the stability group of the spin plane $\gamma_{21}$. This is a nontrivial and beautiful result which can called inversion formula. In Section 4, we introduce a generalized Maxwell equation, and in Section 5, we introduce the generalized Hertz equation. In Section 6, we prove a mathematical Dirac-Maxwell equivalence of the first kind [2, 35], thereby deriving a Dirac-Hestenes equation from the free Maxwell equations. In Section 7, we introduce a new form of a mathematical Maxwell-Dirac equivalence (called MDE of the second kind) different from the one studied in Section 6. This new MDE of the second kind suggests that the electron is a "composite" system. To prove the MDE of the second kind, we decompose a Dirac-Hestenes spinor field satisfying a Dirac-Hestenes equation 
in such a way that it results in a nonlinear generalized Maxwell-like equation (see (7.1)) satisfied by a certain Hertz potential field, mathematically represented by an object of the same mathematical nature as an electromagnetic field, that is, $\Pi \in \sec \bigwedge^{2}(M) \subset \sec \mathscr{b} \ell(M)$. This new equivalence is very suggestive in view of the fact that there are recent (wild) speculations that the electron can be split into two components [6] (see also [38]). If this fantastic claim announced by Maris [21] is true, it is necessary to understand what is going on. The new MDE presented in Section 6 may eventually be useful to understand the mechanism behind the "electron splitting" into electrinos. We are not going to discuss these ideas here. Instead, we concentrate our attention in showing in Section 8 that the analogous on Minkowski spacetime of the famous Seiberg-Witten monopole equations arises naturally from the MDE of the first kind once a reasonable hypothesis is imposed. We also present a possible coherent interpretation of those equations. Indeed, we prove that when the Dirac-Hestenes spinor field satisfying the first of Seiberg-Witten equations is an eigenvector of the parity operator, then that equation describes a pair of massless monopoles of opposite magnetic-like charges, coupled together by its interaction electromagnetic field. Finally, in Section 9, we present our conclusions.

2. Clifford and spin-Clifford bundles. Let $\mathcal{M}=(M, g, D)$ be Minkowski spacetime. The pair $(M, g)$ is a four-dimensional, time-oriented, and space-oriented Lorentzian manifold, with $M \simeq \mathbb{R}^{4}$ and $g \in \sec T^{0,2} M$ being a Lorentzian metric of signature $(1,3)$. Let $T^{*} M[T M]$ be the cotangent [tangent] bundle. Let $T^{*} M=\cup_{x \in M} T_{x}^{*} M, T M=\cup_{x \in M} T_{x} M$, and $T_{x} M \simeq T_{x}^{*} M \simeq \mathbb{R}^{1,3}$, where $\mathbb{R}^{1,3}$ is the Minkowski vector space. Let $D$ be the Levi-Civita connection of $g$, that is, $D g=0, \mathbf{R}(D)=0$. Also $\mathbf{T}(D)=0, \mathbf{R}$ and $\mathbf{T}$ being, respectively, the torsion and curvature tensors. Now, the Clifford bundle of differential forms $\mathscr{C} \ell(M)$ is the bundle of algebras, that is, $\mathscr{C} \ell(M, g)=\cup_{x \in M} \mathscr{b} \ell\left(T_{x}^{*} M\right)$, where for all $x \in M$, $\mathscr{C} \ell\left(T_{x}^{*} M\right)=\mathscr{C}_{1,3}$, the so-called spacetime algebra. Recall also that $\mathscr{C} \ell(M, g)$ is a vector bundle associated to the orthonormal frame bundle, that is, $\mathscr{C} \ell(M, g)$ $=P_{\mathrm{SO}_{+(1,3)}} \times_{a d} \mathscr{C} \ell_{1,3}[22,26]$. For any $x \in M, \mathscr{C} \ell\left(T_{x}^{*} M\right)$ is a linear space over the real field $\mathbb{R}$. Moreover, $\mathscr{C} \ell\left(T_{x}^{*} M\right)$ is isomorphic to the Cartan algebra $\wedge\left(T_{x}^{*} M\right)$ of the cotangent space and $\Lambda\left(T_{x}^{*} M\right)=\sum_{k=0}^{4} \Lambda^{k}\left(T_{x}^{*} M\right)$, where $\Lambda^{k}\left(T_{x}^{*} M\right)$ is the $\left(\begin{array}{l}4 \\ k\end{array}\right)$-dimensional space of $k$-forms. Then, sections of $\mathscr{C} \ell(M, g)$ can be represented as a sum of nonhomogeneous differential forms. Let $\left\langle x^{\mu}\right\rangle$ be Lorentz coordinate functions for $M$ and let $\left\{e_{\mu}\right\} \in \sec F M$ (the frame bundle) be an orthonormal basis for $T M$, that is, $g\left(e_{\mu}, e_{v}\right)=\eta_{\mu \nu}=\operatorname{diag}(1,-1,-1,-1)$. Let $\gamma^{\nu}=d x^{\nu} \in \sec \bigwedge^{1}(M) \subset \sec \mathscr{C} \ell(M, g)(\nu=0,1,2,3)$ such that the set $\left\{\gamma^{\nu}\right\}$ is the dual basis of $\left\{e_{\mu}\right\}$. Moreover, we denote by $\check{g}$ the metric in the cotangent bundle.

2.1. Clifford product. The fundamental Clifford product (in what follows to be denoted by juxtaposition of symbols) is generated by $\gamma^{\mu} \gamma^{v}+\gamma^{v} \gamma^{\mu}=2 \eta^{\mu v}$ 
and if $\mathscr{b} \in \sec \mathscr{b} \ell(M, g)$, we have

$$
\mathscr{C}=s+v_{\mu} \gamma^{\mu}+\frac{1}{2 !} b_{\mu \nu} \gamma^{\mu} \gamma^{\nu}+\frac{1}{3 !} a_{\mu \nu \rho} \gamma^{\mu} \gamma^{\nu} \gamma^{\rho}+p \gamma^{5},
$$

where $\gamma^{5}=\gamma^{0} \gamma^{1} \gamma^{2} \gamma^{3}=d x^{0} d x^{1} d x^{2} d x^{3}$ is the volume element and $s, v_{\mu}, b_{\mu v}$, $a_{\mu v \rho}$, and $p \in \sec \bigwedge^{0}(M) \subset \sec \mathscr{b} \ell(M, g)$.

Let $A_{r} \in \sec \bigwedge^{r}(M)$ and $B_{s} \in \sec \bigwedge^{s}(M)$. For $r=s=1$, we define the scalar product as follows:

$$
a \cdot b=\frac{1}{2}(a b+b a)=\check{g}(a, b)
$$

for $a, b \in \sec \bigwedge^{1}(M) \subset \sec \mathscr{b} \ell(M, g)$. We define also the exterior product $(\forall r, s=$ $0,1,2,3)$ by

$$
A_{r} \wedge B_{s}=\left\langle A_{r} B_{s}\right\rangle_{r+s}, \quad A_{r} \wedge B_{s}=(-1)^{r s} B_{s} \wedge A_{r}
$$

where \langle\rangle$_{k}$ is the component in $\Lambda^{k}(M)$ of the Clifford field. The exterior product is extended by linearity to all sections of $\mathscr{b} \ell(M, g)$.

For $A_{r}=a_{1} \wedge \cdots \wedge a_{r}, B_{r}=b_{1} \wedge \cdots \wedge b_{r}$, the scalar product is defined here as follows:

$$
A_{r} \cdot B_{r}=\left(a_{1} \wedge \cdots \wedge a_{r}\right) \cdot\left(b_{1} \wedge \cdots \wedge b_{r}\right)=\left|\begin{array}{ccc}
a_{1} \cdot b_{1} & \cdots & a_{1} \cdot b_{r} \\
\vdots & \vdots & \vdots \\
a_{r} \cdot b_{1} & \cdots & a_{r} \cdot b_{r}
\end{array}\right| .
$$

We agree that if $r=s=0$, the scalar product is simply the ordinary product in the real field.

Also, if $r \neq s$, then $A_{r} \cdot B_{s}=0$. Finally, the scalar product is extended by linearity to all sections of $\mathscr{C} \ell(M, g)$.

For $r \leq s, A_{r}=a_{1} \wedge \cdots \wedge a_{r}, B_{s}=b_{1} \wedge \cdots \wedge b_{s}$, we define the left contraction by

$$
\begin{aligned}
\lrcorner:\left(A_{r}, B_{S}\right) \longmapsto A_{r}, B_{S}= & \sum_{i_{1}<\cdots<i_{r}} \epsilon^{i_{1} \cdots i_{s}}\left(a_{1} \wedge \cdots \wedge a_{r}\right) \\
& \cdot\left(b_{i_{1}} \wedge \cdots \wedge b_{i_{s}}\right)^{\sim} b_{i_{r}+1} \wedge \cdots \wedge b_{i_{s}},
\end{aligned}
$$

where $\sim$ is the reverse mapping (reversion) defined by

$$
\sim: \sec \bigwedge^{p}(M) \ni a_{1} \wedge \cdots \wedge a_{p} \longmapsto a_{p} \wedge \cdots \wedge a_{1}
$$

and extended by linearity to all sections of $\mathscr{b} \ell(M, g)$. We agree that for $\alpha, \beta \in$ $\sec \wedge^{0}(M)$, the contraction is the ordinary (pointwise) product in the real field 
and that if $\alpha \in \sec \bigwedge^{0}(M), A_{r} \in \sec \bigwedge^{r}(M)$, and $B_{s} \in \sec \bigwedge^{s}(M)$, then $\left(\alpha A_{r}\right), B_{s}=$ $A_{r}\left(\alpha B_{s}\right)$. The left contraction is extended by linearity to all pairs of elements of sections of $\mathscr{b} \ell(M, g)$, that is, for $A, B \in \sec \mathscr{\ell}(M, g)$,

$$
A_{\lrcorner} B=\sum_{r, s}\langle A\rangle_{r}\langle B\rangle_{s}, \quad r \leq s .
$$

It is also necessary to introduce the operator of the right contraction denoted by ${ }_{\llcorner}$. The definition is obtained from the one presenting the left contraction with the imposition that $r \geq s$ and taking into account that now if $A_{r} \in \sec \bigwedge^{r}(M), B_{s} \in \sec \bigwedge^{s}(M)$, then $A_{r\llcorner}\left(\alpha B_{s}\right)=\left(\alpha A_{r}\right)_{\llcorner} B_{s}$.

The main formulas used in the Clifford calculus can be obtained from the following ones (where $a \in \sec \bigwedge^{1}(M) \subset \sec \mathscr{C} \ell(M, g)$ ):

$$
\begin{aligned}
a B_{s} & =a_{\lrcorner} B_{s}+a \wedge B_{s}, \quad B_{s} a=B_{S\llcorner} a+B_{s} \wedge a, \\
a_{\lrcorner} B_{s} & =\frac{1}{2}\left(a B_{s}-(-)^{s} B_{s} a\right), \\
A_{r\lrcorner} B_{s} & =(-)^{r(s-1)} B_{s\llcorner} A_{r}, \\
a \wedge B_{s} & =\frac{1}{2}\left(a B_{s}+(-)^{s} B_{s} a\right), \\
A_{r} B_{s} & =\left\langle A_{r} B_{s}\right\rangle_{|r-s|}+\left\langle A_{r\lrcorner} B_{s}\right\rangle_{|r-s-2|}+\cdots+\left\langle A_{r} B_{s}\right\rangle_{|r+s|} \\
& =\sum_{k=0}^{m}\left\langle A_{r} B_{s}\right\rangle_{|r-s|+2 k} .
\end{aligned}
$$

2.1.1. Hodge star operator. Let $\star$ be the Hodge star operator, that is, the mapping

$$
\star: \bigwedge^{k}(M) \longrightarrow \bigwedge^{4-k}(M), \quad A_{k} \longmapsto \star A_{k},
$$

where for $A_{k} \in \sec \bigwedge^{k}(M) \subset \sec \mathscr{b} \ell(M, g)$,

$$
\left[B_{k} \cdot A_{k}\right] \tau_{g}=B_{k} \wedge \star A_{k}, \quad \forall B_{k} \in \sec \bigwedge^{k}(M) \subset \sec \mathscr{b} \ell(M) .
$$

In (2.10), $\tau_{\mathfrak{g}} \in \Lambda^{4}(M)$ is a standard volume element. Then we can verify that

$$
\star A_{k}=\tilde{A}_{k} \gamma^{5} .
$$

2.1.2. Dirac operator. Let $d$ and $\delta$ be, respectively, the differential and Hodge codifferential operators acting on sections of $\bigwedge(M)$. If $A_{p} \in \sec \bigwedge^{p}(M) \subset$ $\sec \mathscr{C} \ell(M)$, then $\delta A_{p}=(-)^{p} \star^{-1} d \star A_{p}$, with $\star^{-1} \star=$ identity.

The Dirac operator acting on sections of $\mathscr{b} \ell(M, g)$ is the invariant first-order differential operator

$$
\partial=\gamma^{a} D_{e_{a}},
$$


where $\left\{e_{a}\right\}$ is an arbitrary orthonormal basis for $T U \subset T M$ and $\left\{\gamma_{b}\right\}$ is a basis for $T^{*} U \subset T^{*} M$ dual to the basis $\left\{e_{a}\right\}$, that is, $\gamma^{b}\left(e_{a}\right)=\delta_{b}^{a}, a, b=0,1,2,3$. The reciprocal basis of $\left\{\gamma^{b}\right\}$ is denoted by $\left\{\gamma_{a}\right\}$ and we have $\gamma_{a} \cdot \gamma_{b}=\eta_{a b}$ $\left(\eta_{a b}=\operatorname{diag}(1,-1,-1,-1)\right)$. Also,

$$
D_{e_{a}} \gamma^{b}=-\omega_{a}^{b c} \gamma_{c}
$$

Defining

$$
\omega_{a}=\frac{1}{2} \omega_{a}^{b c} \gamma_{b} \wedge \gamma_{c},
$$

we have that for any $A_{p} \in \sec \bigwedge^{p}(M), p=0,1,2,3,4$,

$$
D_{e_{a}} A=e_{a}+\frac{1}{2}\left[\omega_{a}, A\right]
$$

Using (2.15), we can show the very important result

$$
\begin{gathered}
\left.\partial A_{p}=\partial \wedge A_{p}+\partial\right\lrcorner A_{p}=d A_{p}-\delta A_{p}, \\
\partial \wedge A_{p}=d A_{p}, \quad \partial_{\lrcorner} A_{p}=-\delta A_{p} .
\end{gathered}
$$

2.2. Dirac-Hestenes spinor fields. Now, as is well known, an electromagnetic field is represented by $F \in \sec \bigwedge^{2}(M) \subset \sec \mathscr{b} \ell(M, g)$. How can we represent the Dirac spinor fields in this formalism? We can show that Dirac-Hestenes spinor fields do the job. We give here a short introduction to these objects (when living on Minkowski spacetime) which serves mainly the purpose of fixing notations. For a rigorous theory of these objects (using vector bundles) on a general Riemann-Cartan manifold see [22]. Recall that there is a $2: 1$ mapping $\mathbf{s}^{\prime}: \Theta^{\prime} \rightarrow \mathscr{B}$ between $\mathscr{B}$, the set of all orthonormal ordered vector frames, and $\Theta^{\prime}$, the set of all spin frames of $T^{*} M$. As discussed at length in [22, 26], a spin coframe can be thought of as a basis of $T^{*} M$ such that two ordered bases, even if consisting of the same vectors but with the spatial vectors differing by a $2 \pi$ rotation, are considered distinct, and two ordered basis, even if consisting of the same vectors but with the spatial vectors differing by a $4 \pi$ rotation, are identified. For short, in this paper, we simply call the spin coframes spin frames. Also, vector coframes are simply called vector frames in what follows.

Consider the set $\mathscr{S}$ of mappings

$$
M \ni x \longmapsto u(x) \in \operatorname{Spin}_{+}(1,3) \simeq \mathrm{SL}(2, \mathbb{C})
$$

Choose a constant spin frame $\left\{\gamma_{a}\right\} \in \mathscr{B}, a=0,1,2,3$, and choose $\Xi_{0} \in \Theta^{\prime}$ corresponding to a constant mapping $u_{0} \in \mathscr{Y}$. By constant we mean that the equation $\gamma_{\mu}(x)=\gamma_{\mu}(y)\left((\mu=0,1,2,3)\right.$ and $\left.u_{0}(x)=u_{0}(y), \forall x, y \in M\right)$ has meaning due to the usual affine structure that can be given to Minkowski spacetime. $\Xi_{0}, \Xi_{u} \in \boldsymbol{\Theta}^{\prime}$ are related as follows:

$$
u_{0} \mathbf{s}^{\prime}\left(\Xi_{0}\right) u_{0}^{-1}=u \mathbf{s}^{\prime}\left(\Xi_{u}\right) u^{-1}
$$


From now on, in order to simplify the notation, we take $u_{0}=1$. The frame $\mathbf{s}^{\prime}\left(\Xi_{0}\right)=\left\{\gamma_{a}\right\}$ is called the fiducial vector frame and $\Xi_{0}$ the fiducial spin frame. We note that (2.18) is satisfied by two such $u$ 's differing by a signal, and, of course, $\mathbf{s}^{\prime}\left(\Xi_{u}\right)=\mathbf{s}^{\prime}\left(\Xi_{-u}\right)$.

Let

$$
\mathbb{C}=\left\{\left(\Xi_{u}, \Psi_{\Sigma_{u}}\right) \mid u \in \mathscr{S}, \Xi_{u} \in \Theta^{\prime}, \Psi_{\Xi_{u}} \in \sec \bigwedge^{+} M \subset \sec \mathscr{b} \ell^{+}(M, g)\right\},
$$

where $\bigwedge^{+} M=\bigwedge^{0} M+\bigwedge^{2} M+\bigwedge^{4} M$.

We define an equivalence relation on $\mathbb{t}$ by setting

$$
\left(\Xi_{u}, \Psi_{\Xi_{u}}\right) \sim\left(\Xi_{u^{\prime}}, \Psi_{\Xi_{u^{\prime}}}\right)
$$

if and only if

$$
u \mathbf{s}^{\prime}\left(\Xi_{u}\right) u^{-1}=u^{\prime-1} \mathbf{s}^{\prime}\left(\Xi_{u^{\prime}}\right) u^{\prime}, \quad \Psi_{\Xi_{u^{\prime}}}=\Psi_{\Xi_{u}} u u^{\prime-1} .
$$

DEFINITION 2.1. Any equivalence class $\left[\left(\Xi_{u}, \Psi_{\Xi_{u}}\right)\right]$ will be called a DiracHestenes spinor field (DHSF).

Before proceeding, we recall that a more rigorous definition of a DHSF as a section of a spin-Clifford bundle is given in [22]. We will not need such a sophistication in what follows.

Note that the pairs $\left(\Xi_{u}, \Psi_{\Xi_{u}}\right)$ and $\left(\Xi_{-u},-\Psi_{\Sigma_{-u}}\right)$ are equivalent but the pairs $\left(\Xi_{u}, \Psi_{\Sigma_{u}}\right)$ and $\left(\Xi_{-u}, \Psi_{\Xi_{-u}}\right)$ are not. This distinction is essential in order to give a structure of linear space (over the real numbers) to the set $\mathscr{T}$. Indeed, such a linear structure on $\mathcal{T}$ is defined as follows:

$$
\begin{aligned}
a\left[\left(\Xi_{u_{1}}, \Psi_{\Xi_{u_{1}}}\right)\right]+b\left[\left(\Xi_{u_{2}}, \Psi_{\Xi_{u_{2}}}\right)\right] & =\left[\left(\Xi_{u_{1}}, a \Psi_{\Xi_{u_{1}}}\right)\right]+\left[\left(\Xi_{u_{2}}, b \Psi_{\Xi_{u_{2}}}\right)\right], \\
(a+b)\left[\left(\Xi_{u_{1}}, \Psi_{\Xi_{u_{1}}}\right)\right] & =a\left[\left(\Xi_{u_{1}}, \Psi_{\Xi_{u_{1}}}\right)\right]+b\left[\left(\Xi_{u_{1}}, \Psi_{\Xi_{u_{1}}}\right)\right], \quad a, b \in \mathbb{R} .
\end{aligned}
$$

We can simplify the notation by recalling that every $u \in \mathscr{S}$ determines, of course, a unique spin frame $\Xi_{u}$. Taking this into account, we consider the set of all pairs $\left(u, \Psi_{\Xi_{u}}\right) \in \mathscr{S} \times \sec \mathscr{b} \ell^{+}(M, g)$.

We define an equivalence relation $\mathscr{R}$ in $\mathscr{S} \times \sec \mathscr{C} \ell^{+}(M, g)$ as follows. Two pairs $\left(u, \Psi_{\Xi_{u}}\right),\left(u^{\prime}, \Psi_{\Xi_{u^{\prime}}}\right) \in \sec \mathscr{Y} \times \sec \mathscr{b} \ell^{+}(M, g)$ are equivalent if and only if

$$
\Psi_{\Xi_{u^{\prime}}} u^{\prime}=\Psi_{\Xi_{u}} u
$$

Of course, $\mathbf{s}^{\prime}\left(\Xi_{u^{\prime}}\right)=v \mathbf{s}^{\prime}\left(\Xi_{u}\right) v^{-1}$ with $v=\left(u^{\prime}\right)^{-1} u \in \mathscr{Y}$. Note that the pairs $\left(u, \Psi_{\Xi_{u}}\right)$ and $\left(-u,-\Psi_{\Xi_{u}}\right)$ are equivalent but the pairs $\left(u, \Psi_{\Xi_{u}}\right)$ and $\left(-u, \Psi_{\Xi_{u}}\right)$ are not. 
Denote by $\mathscr{S} \times \sec \mathscr{b} \ell^{+}(M, g) / \mathscr{R}$ the quotient set of the equivalence classes generated by $\mathscr{R}$. Their elements are called Dirac-Hestenes spinors. Of course this is the same definition as above.

From now on, we simplify even more our notation. In this way, if we take two orthonormal spin frames $\mathbf{s}^{\prime}(\Xi)=\left\{\gamma^{\mu}\right\}$ and $\mathbf{s}^{\prime}(\dot{\Xi})=\left\{\dot{\gamma}^{\mu}=R \gamma^{\mu} \widetilde{R}=\Lambda_{v}^{\mu} \gamma^{\nu}\right\}$ with $\Lambda_{v}^{\mu} \in \mathrm{SO}^{e}(1,3)$ and $R(x) \in \operatorname{Spin}^{e}(1,3)$ for all $x \in M, R \widetilde{R}=\widetilde{R} R=1$, then we simply write the relation (2.23) between representatives of a Dirac-Hestenes spinor field in the two spin frames as the sections $\psi_{\Xi}$ and $\psi_{\dot{\Xi}}$ of $\mathscr{b} \ell^{+}(M, g)$ related by

$$
\psi_{\dot{\Xi}}=\psi_{\Xi} R
$$

Recall that since $\psi_{\Xi} \in \sec \stackrel{+}{\wedge} M \subset \sec ^{C} \mathscr{C} \ell^{+}(M, g)$, we have

$$
\psi_{\Xi}=s+\frac{1}{2 !} b_{\mu \nu} \gamma^{\mu} \gamma^{\nu}+p \gamma^{5} .
$$

Note that $\psi_{\Xi}$ has the correct number of degrees of freedom in order to represent a Dirac spinor field and recall that the specification of $\psi_{\Xi}$ depends on the spin frame $\Xi$. To simplify even more our notation, when it is clear which is the spin frame $\Xi$, and no possibility of confusion arises, we simply write $\psi$ instead of $\psi_{\Xi}$.

When $\psi \tilde{\psi} \neq 0$, where $\sim$ is the reversion operator, we can show that $\psi$ has the following canonical decomposition:

$$
\psi=\sqrt{\rho} e^{\beta \gamma_{5} / 2} R
$$

where $\rho, \beta \in \sec \bigwedge^{0}(M) \subset \sec \mathscr{b} \ell(M, g)$ and $R(x) \in \operatorname{Spin}^{e}(1,3) \subset \mathscr{C}_{1,3}^{+}$, for all $x \in M$. The function $\beta$ is called the Takabayasi angle. If we want to work in terms of the usual Dirac spinor field formalism, we can translate our results by choosing, for example, the standard matrix representation of the 1-form $\left\{\gamma^{\mu}\right\}$ in $\mathbb{C}(4)$ (the algebra of the complex $4 \times 4$ matrices), and for $\psi_{\Sigma}$ given by (2.14), we have the following (standard) matrix representation [26, 30]:

$$
\Psi=\left(\begin{array}{rrrr}
\psi_{1} & -\psi_{2}^{*} & \psi_{3} & \psi_{4}^{*} \\
\psi_{2} & \psi_{1}^{*} & \psi_{4} & -\psi_{3}^{*} \\
\psi_{3} & \psi_{4}^{*} & \psi_{1} & -\psi_{2}^{\star} \\
\psi_{4} & -\psi_{3}^{*} & \psi_{2} & \psi_{1}^{\star}
\end{array}\right),
$$

where $\psi_{k}(x) \in \mathbb{C}, k=1,2,3,4$, and for all $x \in M$.

We recall that a standard Dirac spinor field is a section $\Psi_{D}$ of the vector bundle $P_{\text {Spin }^{e}(1,3)} \times_{\lambda} \mathbb{C}(4)$, where $\lambda$ is the $D(1 / 2,0) \oplus D(0,1 / 2)$ representation of $\operatorname{SL}(2, \mathbb{C}) \sim \operatorname{Spin}^{e}(1,3)$. For details see, for example, [22, 26]. The relation 
between $\Psi_{D}$ and $\psi$ is given by

$$
\Psi_{D}=\left(\begin{array}{c}
\psi_{1} \\
\psi_{2} \\
\psi_{3} \\
\psi_{4}
\end{array}\right)=\left(\begin{array}{c}
s-i b_{12} \\
-b_{13}-i b_{23} \\
-b_{03}+i p \\
-b_{01}-i b_{02}
\end{array}\right),
$$

where $s, b_{12}, \ldots$ are the real functions in (2.25) and $*$ denotes the complex conjugation.

We recall that the even subbundle $\mathscr{b} \ell^{+}(M, g)$ of $\mathscr{b} \ell(M, g)$ is such that its typical fiber is the Pauli algebra $\mathscr{C} \ell_{3,0} \equiv \mathscr{C} \ell_{1,3}^{+}$(which is isomorphic to $\mathbb{C}$ (2), the algebra of $2 \times 2$ complex matrices). Elements of $\mathscr{b} \ell_{1,3}^{+}$are called biquaternions in the old literature. The isomorphism $\mathscr{C}_{3,0} \equiv \mathscr{C} \ell_{1,3}^{+}$is exhibited by putting $\vec{\sigma}_{i}=\gamma_{i} \gamma_{0}$, whence $\vec{\sigma}_{i} \vec{\sigma}_{j}+\vec{\sigma}_{j} \vec{\sigma}_{i}=2 \delta_{i j}$. We recall also that the Dirac algebra is $\mathscr{C} \ell_{4,1} \equiv \mathbb{C}(4)$ and $\mathscr{C} \ell_{4,1} \equiv \mathbb{C} \otimes \mathscr{C}_{1,3}$.

Consider the complexification $\mathscr{b} \ell_{C}(M, g)$ of $\mathscr{b} \ell(g)$ called the complex Clifford bundle. Then $\mathscr{C}_{C}(M, g)=\mathbb{C} \otimes \mathscr{b} \ell(M, g)$, and we can verify that the typical fiber of $\mathscr{C} \ell_{C}(M, g)$ is $\mathscr{C} \ell_{4,1}=\mathbb{C} \otimes \mathscr{C} \ell_{1,3}$, the Dirac algebra. Now let $\left\{\Delta_{0}, \Delta_{1}, \Delta_{2}, \Delta_{3}, \Delta_{4}\right\}$ $\subset \sec \mathscr{b} \ell_{C}(M, g)$ be, for all $x \in M$, an orthonormal basis of $\mathfrak{C}_{4,1}$. We have

$$
\Delta_{a} \Delta_{b}+\Delta_{b} \Delta_{a}=2 g_{a b}, \quad g_{a b}=\operatorname{diag}(+1,+1,+1,+1,-1) .
$$

We identify $\gamma_{\mu}=\Delta_{\mu} \Delta_{4}$ and call $I=\Delta_{0} \Delta_{1} \Delta_{2} \Delta_{3} \Delta_{4}$. Since $I^{2}=-1$ and $I$ commutes with all elements of $\mathscr{C}_{4,1}$, we identify $I$ with $i=\sqrt{-1}$ and $\gamma_{\mu}$ with a fundamental set generating the local Clifford algebra of $\mathscr{b} \ell(M, g)$. Then if $\mathscr{A} \in \sec \ell_{C}(M, g)$, we have

$$
\mathscr{A}=\Phi_{s}+A_{C}^{\mu} \gamma_{\mu}+\frac{1}{2} B_{C}^{\mu v} \gamma_{\mu} \gamma_{v}+\frac{1}{3 !} \tau_{C}^{\mu v \rho} \gamma_{\mu} \gamma_{v} \gamma_{v}+\Phi_{p} \gamma_{5}
$$

where $\Phi_{s}, \Phi_{p}, A_{C}^{\mu}, B_{C}^{\mu \nu}, \tau_{C}^{\mu \nu \rho} \in \sec \mathbb{C} \otimes \Lambda^{0}(M) \subset \sec \mathscr{C} \ell_{C}(M, g)$, that is, for all $x \in$ $M, \Phi_{s}(x), \Phi_{p}(x), A_{C}^{\mu}(x), B_{C}^{\mu v}(x)$, and $\tau_{C}^{\mu v \rho}(x)$ are complex numbers.

Now, it can be verified that

$$
f=\frac{1}{2}\left(1+\gamma_{0}\right) \frac{1}{2}\left(1+i \gamma_{1} \gamma_{2}\right), \quad f^{2}=f,
$$

is a primitive idempotent field of $\mathscr{C}_{C}(M, g)$. We can also verify without difficulty that if $=\gamma_{2} \gamma_{1} f$.

Appropriate equivalence classes (see $[22,26])$ of $\mathscr{C}_{C}(M, g) f$ are representatives of the standard Dirac spinor fields in $\mathscr{C}_{C}(M, g)$. We can easily show that the representation of $\Psi_{D}$ in $\mathscr{C}_{C}(M, g)$ is given by

$$
\Psi_{D}=\psi f
$$

where $\psi$ is the Dirac-Hestenes spinor field given by (2.25). 
2.3. Weyl spinors and parity operator. By definition, $\psi \in \sec \mathscr{b} \ell^{+}(M, g)$ is a representative of a Weyl spinor field $[17,18]$ if, besides being a representative of a Dirac-Hestenes spinor field, it satisfies $\gamma_{5} \psi= \pm \psi \gamma$, where

$$
\gamma_{21}=\gamma_{2} \gamma_{1}
$$

The positive (negative) "eigenstates" of $\gamma_{5}$ will be denoted by $\psi_{+}\left(\psi_{-}\right)$. For a general $\psi \in \sec \mathscr{C} \ell^{+}(M, g)$, we can write

$$
\psi_{ \pm}=\frac{1}{2}\left[\psi \mp \gamma_{5} \psi \gamma_{21}\right] .
$$

Then

$$
\psi=\psi_{+}+\psi_{-} .
$$

The parity operator $\mathbf{P}$ in our formalism is represented in such a way that for $\psi \in \sec ^{C} \ell^{+}(M, g)$,

$$
\mathbf{P} \psi=-\gamma_{0} \psi \gamma_{0}
$$

The following Dirac-Hestenes spinor fields are eigenstates of the parity operator with eigenvalues \pm 1 :

$$
\begin{array}{ll}
\mathbf{P} \psi^{\dagger}=+\psi^{\uparrow}, & \psi^{\uparrow}=\gamma_{0} \psi_{-} \gamma_{0}-\psi_{-}, \\
\mathbf{P} \psi^{\downarrow}=-\psi^{\downarrow}, & \psi^{\downarrow}=\gamma_{0} \psi_{+} \gamma_{0}+\psi_{+} .
\end{array}
$$

2.4. The spin-Dirac operator. Associated with the covariant derivative operator $D_{e_{a}}$ (see (2.13)) acting on sections of the Clifford bundle, there is a spincovariant derivative operator $D_{e_{a}}^{s}$ acting on sections of a right spin-Clifford bundle such that they are Dirac-Hestenes spinor fields. Hopefully, it will not be necessary to present the details concerning this concept here (see [22]). It is enough to say that $D_{e_{a}}^{s}$ has a representative on the Clifford bundle, called $D_{e_{a}}^{(s)}$, such that if $\psi_{\Xi}$ is a representative of a Dirac-Hestenes spinor field, we have

$$
D_{e_{a}}^{(s)} \psi_{\Xi}=e_{a}\left(\psi_{\Xi}\right)+\frac{1}{2} \omega_{a} \psi_{\Xi},
$$

where $\boldsymbol{\omega}_{a}$ has been defined by (2.14). The representative of the spin-Dirac operator acting on representatives of Dirac-Hestenes spinor fields is the invariant first-order operator given by

$$
\partial^{(s)}=\gamma^{a} D_{e_{a}}^{(s)} .
$$

From the definition of the spin Dirac operator, we see that if we restrict our considerations to orthonormal coordinate basis $\left\{\gamma^{\mu}=d x^{\mu}\right\}$, where $\left\{x^{\mu}\right\}$ are global Lorentz coordinates, then $\boldsymbol{\omega}_{\mu}=0$ and the action of $\partial^{(s)}$ on DiracHestenes spinor fields is the same as the action of $\partial$ on these fields. 
2.5. Maxwell and Dirac-Hestenes equations. With the mathematical tools presented above, we have the Maxwell equation

$$
\partial F=J_{e}
$$

satisfied by an electromagnetic field $F \in \sec \bigwedge^{2}(M) \subset \sec \mathscr{b} \ell(M, g)$, and generated by a current $J_{e} \in \sec \bigwedge^{1}(M) \subset \sec \mathscr{b} \ell(M, g)$.

The Dirac-Hestenes equation in a spin frame $\Xi$ satisfied by a Dirac-Hestenes spinor field $\psi \in \sec \left[\bigwedge^{0}(M)+\bigwedge^{2}(M)+\Lambda^{4}(M)\right] \subset \sec \mathscr{b} \ell(M, g)$ is

$$
\partial \psi \gamma^{2} \gamma^{1}-m \psi \gamma^{0}+\frac{1}{2} \gamma^{a} \psi \omega_{a} \gamma^{2} \gamma^{1}=0 .
$$

For what follows, we restrict our considerations only to the case of orthonormal coordinate basis, in which case the Dirac-Hestenes equation reads

$$
\partial \psi \gamma^{2} \gamma^{1}-m \psi \gamma^{0}=0 .
$$

3. Solution of $\psi \gamma_{21} \tilde{\psi}=F$. We now want to solve (1.1) for $\psi$. Before proceeding, we observe that on Euclidean spacetime this equation has been solved using Clifford algebra methods in [15]. Also, on Minkowski spacetime, a particular solution of an equivalent equation (written in terms of biquaternions) appears in [7]. We are going to show that, contrary to the claims of [14], a general solution for $\psi$ has indeed eight degrees of freedom, although two of them are arbitrary, that is, not fixed by $F$ alone. Once we give a solution of (1.1) for $\psi$, the reason for the indetermination of two of the degrees of freedom will become clear. This involves the Fierz identities, boomerangs [17, 27, 30], and the general theorem permitting the reconstruction of spinors from their bilinear covariants.

We start by observing that from (1.1) and (2.26), we can write

$$
F=\rho e^{\beta \gamma_{5}} R \gamma_{21} \tilde{R} .
$$

Then, defining $f=F / \rho e^{\beta \gamma_{5}}$, it follows that

$$
\begin{aligned}
f & =R \gamma_{21} \tilde{R}, \\
f^{2} & =-1 .
\end{aligned}
$$

Now, since all objects in (3.1) and (3.2) are even, we can take the advantage of the isomorphism $\mathscr{C}_{3,0} \equiv \mathscr{C}_{1,3}^{+}$and make the calculations when convenient in the Pauli algebra. To this end, we first write

$$
F=\frac{1}{2} F^{\mu v} \gamma_{\mu} \gamma_{\nu}, \quad F^{\mu \nu}=\left(\begin{array}{cccc}
0 & -E^{1} & -E^{2} & -E^{3} \\
E^{1} & 0 & -B^{3} & B^{2} \\
E^{2} & B^{3} & 0 & -B^{1} \\
E^{3} & -B^{2} & B^{1} & 0
\end{array}\right),
$$


where $\left(E^{1}, E^{2}, E^{3}\right)$ and $\left(B^{1}, B^{2}, B^{3}\right)$ are, respectively, the Cartesian components of the electric and magnetic fields.

We now write $F$ in $\mathscr{C} \ell^{+}(M, g)$, the even subalgebra of $\mathscr{b} \ell(M, g)$. The typical fiber of $\mathscr{C} \ell^{+}(M, g)$ (which is also a vector bundle) is isomorphic to the Pauli algebra. We put

$$
\vec{\sigma}_{i}=\gamma_{i} \gamma_{0}, \quad \mathbf{i}=\vec{\sigma}_{1} \vec{\sigma}_{2} \vec{\sigma}_{3}=\gamma_{0} \gamma_{1} \gamma_{2} \gamma_{3}=\gamma_{5}
$$

Recall that $\mathbf{i}$ commutes with bivectors and, since $\mathbf{i}^{2}=-1$, it acts like the imaginary unit $i=\sqrt{-1}$ in $\mathscr{b} \ell^{+}(M, g)$. From (3.4) and (3.5) (taking into account our previous discussion), we can write

$$
F=\vec{E}+\mathbf{i} \vec{B}
$$

with $\vec{E}=E^{i} \vec{\sigma}_{i}, \vec{B}=B^{j} \vec{\sigma}_{j}, i, j=1,2,3$. We can write an analogous equation for $f:$

$$
f=\vec{e}+\mathbf{i} \vec{b}
$$

Now, since $F^{2} \neq 0$ and

$$
F^{2}=F \cdot F+F \wedge F=-\left(\vec{E}^{2}-\vec{B}^{2}\right)+2 \mathbf{i}(\vec{E} \cdot \vec{B})
$$

the above equations give (in the more general case where both $I_{1}=\left(\vec{E}^{2}-\vec{B}^{2}\right) \neq 0$ and $\left.I_{2}=(\vec{E} \cdot \vec{B}) \neq 0\right)$

$$
\rho=\frac{\sqrt{\vec{E}^{2}-\vec{B}^{2}}}{\cos [\operatorname{arctg} 2 \beta]}, \quad \beta=\frac{1}{2} \arctan \left(\frac{2(\vec{E} \cdot \vec{B})}{\vec{E}^{2}-\vec{B}^{2}}\right) .
$$

Also,

$$
\vec{e}=\frac{1}{\rho}[(\vec{E} \cos \beta+\vec{B} \sin \beta)], \quad \vec{b}=\frac{1}{\rho}[(\vec{B} \cos \beta-\vec{E} \sin \beta)] .
$$

3.1. A particular solution. Now, we can verify that

$$
\begin{aligned}
& L=\frac{\gamma_{21}+f}{\sqrt{2\left(1-\gamma_{5} \mathfrak{I}\right)}}=\frac{\vec{\sigma}_{3}-\mathbf{i} \vec{f}}{\mathbf{i} \sqrt{2\left(1-\mathbf{i}\left(\vec{f} \cdot \vec{\sigma}_{3}\right)\right)}}, \\
& \underline{I}=f^{03}-\gamma_{5} f^{12} \equiv \vec{f} \cdot \vec{\sigma}_{3},
\end{aligned}
$$

is a Lorentz transformation, that is, $L \tilde{L}=\tilde{L} L=1$. Moreover, $L$ is a particular solution of (3.2). Indeed,

$$
\frac{\gamma_{21}+f}{\sqrt{2\left(1-\gamma_{5} \mathfrak{I}\right)}} \gamma_{21} \frac{\gamma_{12}-f}{\sqrt{2\left(1-\gamma_{5} \mathfrak{I}\right)}}=\frac{f\left[2\left(1-\gamma_{5} \mathfrak{I}\right)\right]}{2\left(1-\gamma_{5} \mathfrak{I}\right)}=f .
$$


Of course, since $f^{2}=-1, \vec{e}^{2}=\vec{b}^{2}-1$, and $\vec{e} \cdot \vec{b}=0$, there are only four real degrees of freedom in the Lorentz transformation $L$. From this result in [14], it is concluded that the solution of (1.1) is the Dirac-Hestenes spinor field

$$
\phi=\sqrt{\rho} e^{\gamma_{5} \beta} L,
$$

which has only six degrees of freedom and thus is not equivalent to a general Dirac-Hestenes spinor field (the spinor field that must appear in the DiracHestenes equation), which has eight degrees of freedom. In this way, it is stated in [14] that the MDE of first kind proposed in [35, 39] cannot hold. Although it is true that (3.13) is a solution of (1.1), it is not a general solution, but only a particular one.

Before leaving this section, we mention that there are many other Dirac-like forms of the Maxwell equations published in the literature. All are trivially related in a very simple way and in principle have nothing to do with the two kinds of MDE discussed in the present paper, see [27].

3.2. The general solution. The general solution $R$ of (1.1) is trivially found. It is

$$
R=L S
$$

where $L$ is the particular solution just found and $S$ is any member of the stability group of $\gamma_{21}$, that is,

$$
S \gamma_{21} \tilde{S}=\gamma_{21}, \quad S \tilde{S}=\tilde{S} S=1 .
$$

It is trivial to find that we can parametrize the elements of the stability group as

$$
S=\exp \left(\gamma_{03} \nu\right) \exp \left(\gamma_{21} \varphi\right)
$$

with $0 \leq v<\infty$ and $0 \leq \varphi<\infty$. This shows that the most general DiracHestenes spinor field that solves (1.1) has indeed eight degrees of freedom (as it must be the case, if the claims of [35, 39] are to make sense), although two degrees of freedom are arbitrary, that is, they are like hidden variables!

Now, the reason for the indetermination of two degrees of freedom has to do with a fundamental mathematical result: the fact that a spinor can only be reconstructed through the knowledge of its bilinear covariants and the Fierz identities. Explicitly, to reconstruct a Dirac-Hestenes spinor field $\psi$, it is necessary to know also, besides the bilinear covariant given by (1.1), the following bilinear covariants:

$$
J=\psi \gamma_{0} \tilde{\psi}, \quad K=\psi \gamma_{3} \tilde{\psi} .
$$


Now, $J, K$, and $F$ are related through the so-called Fierz identities:

$$
\begin{aligned}
J^{2} & =-K^{2}=-\sigma^{2}-\omega^{2}, \\
J \cdot K & =0, \quad J \wedge K=-\left(\omega+\gamma_{5}\right) F, \\
\sigma & =\rho \cos \beta, \quad \omega=\rho \sin \beta .
\end{aligned}
$$

In the most general case, when both $\sigma$ and $\omega$ are not 0 , we also have the notable identities first found by Crawford [7] (and which can be derived almost trivially using the Clifford bundle formalism):

$$
\begin{aligned}
& F_{\llcorner} J=\omega K, \quad F_{\llcorner} K=\omega J, \\
& \left(\gamma_{5} F\right)_{\llcorner} J=\sigma K, \quad\left(\gamma_{5} F\right)_{\llcorner} K=\sigma J, \\
& F \cdot F=\omega^{2}-\sigma^{2}, \quad\left(\gamma_{5} F\right) \cdot F=2 \sigma \omega, \\
& J F=-\left(\omega+\gamma_{5} \sigma\right) K, \quad K F=-\left(\omega+\gamma_{5} \sigma\right) J, \\
& F^{2}=\omega^{2}-\sigma^{2}-2 \gamma_{5} \sigma \omega, \quad F^{-1}=K F K /\left(\omega^{2}+\sigma^{2}\right) .
\end{aligned}
$$

Once we know $\omega, \sigma, J, K$, and $F$, we can recover the Dirac-Hestenes spinor field as follows. First, introduce a boomerang $[17,18,30] Z \in \mathscr{C}_{C}(M, g)$ given by

$$
Z=\sigma+J+i F-i K \omega
$$

Then, we can construct $\Psi=Z f \in \mathscr{C}_{C}(M, g) f$ which has the following matrix representation (once the standard representation of the Dirac gamma matrices are used):

$$
\hat{\Psi}=\left(\begin{array}{llll}
\psi_{1} & 0 & 0 & 0 \\
\psi_{2} & 0 & 0 & 0 \\
\psi_{3} & 0 & 0 & 0 \\
\psi_{4} & 0 & 0 & 0
\end{array}\right)
$$

Now, it can easily be verified that $\Psi=Z f$ determines the same bilinear covariants as the ones determined by $\psi$. Note, however, that this spinor is not unique. In fact, $Z$ determines a class of elements $Z \eta$, where $\eta$ is an arbitrary element of $\mathscr{C} \ell_{C}(M, g) f$ which differs one from the other by a complex phase factor $[17,18,30]$.

Recalling that a representative of a Dirac-Hestenes spinor field determines a unique element of $\Phi \in \mathscr{C} \ell_{C}(M) f$ by $\Phi=\psi f$, then it follows (from (3.21) and (2.27) that gives the matrix representation of $\psi$ ) that we can trivially reconstruct an $\psi$ that solves our problem.

4. The generalized Maxwell equation. To comment on the basic error in [14] concerning the Clifford bundle formulation of the generalized Maxwell equation, we recall the following. 
The generalized Maxwell equation [19, 27] which describes the electromagnetic field generated by charges and monopoles can be written in the Cartan bundle as

$$
d F=K_{m}, \quad d G=K_{e},
$$

where $F, G \in \bigwedge^{2}(M)$ and $K_{m}, K_{e} \in \Lambda^{3}(M)$.

These equations are independent of any metric structure defined on the world manifold. When a metric is given and the Hodge dual operator $\star$ is introduced, it is supposed that in vacuum we have $G=\star F$. In this case, putting $K_{e}=-\star J_{e}$ and $K_{m}=\star J_{m}$, with $J_{e}, J_{m} \in \sec \bigwedge^{1}(M)$, we can write the following equivalent set of equations:

$$
\begin{aligned}
d F & =-\star J_{m}, \quad d \star F=-\star J_{e}, \\
\delta(\star F) & =J_{m}, \quad \delta F=-J_{e}, \\
\delta(\star F) & =J_{m}, \quad \delta F=-J_{e}, \\
d F & =-\star J_{m}, \quad \delta F=-J_{e} .
\end{aligned}
$$

Now, supposing that any $\sec \bigwedge^{j}(M) \subset \sec \mathscr{C} \ell(M, g)(j=0,1,2,3,4)$ and taking into account (2.12), (2.13), (2.14), (2.15), and (2.16), we get (1.2) by summing the two equations in (4.5), that is,

$$
(d-\delta) F=J_{e}+K_{m} \quad \text { or } \quad(d-\delta) \star F=-J_{m}+K_{e},
$$

or equivalently

$$
\partial F=J_{e}+\gamma_{5} J_{m} \quad \text { or } \quad \partial\left(-\gamma_{5} F\right)=-J_{m}+\gamma_{5} J_{e} .
$$

Now, writing with the conventions of Section 2,

$$
F=\frac{1}{2} F^{\mu v} \gamma_{\mu} \gamma_{v}, \quad \star F=\frac{1}{2}\left({ }^{\star} F^{\mu v}\right) \gamma_{\mu} \gamma_{v},
$$

then generalized Maxwell equations in the form given by (4.3) can be written in components (in a Lorentz coordinate chart) as

$$
\partial_{\mu} F^{\mu v}=J_{e}^{\mu}, \quad \partial_{\mu}\left({ }^{\star} F^{\mu v}\right)=-J_{m}^{\mu}
$$

Now, assuming as in (1.1) that $F=\psi \gamma_{21} \tilde{\psi}$ and taking into account the relation between $\psi$ and the representation of the standard Dirac spinor $\Psi_{D}$ given by (2.28), we can write (4.9) as

$$
\begin{array}{lc}
\partial_{\mu} \bar{\Psi}_{D}\left[\hat{\gamma}_{\mu}, \hat{\gamma}_{v}\right] \Psi_{D}=2 J_{e}^{\mu}, \quad \partial_{\mu} \bar{\Psi}_{D} \hat{\gamma}_{5}\left[\hat{\gamma}_{\mu}, \hat{\gamma}_{v}\right] \Psi_{D}=-2 J_{m}^{\mu}, \\
F^{\mu v}=\frac{1}{2} \bar{\Psi}_{D}\left[\hat{\gamma}_{\mu}, \hat{\gamma}_{v}\right] \Psi, \quad\left({ }^{\star} F^{\mu v}\right)=\frac{1}{2} \bar{\Psi}_{D} \hat{\gamma}_{5}\left[\hat{\gamma}_{\mu}, \hat{\gamma}_{\nu}\right] \Psi_{D} .
\end{array}
$$


The reverse of the first equation of (4.7) reads

$$
(\tilde{\partial F})=J_{e}-K_{m}
$$

First summing, and then subtracting (1.2) from (4.1) we get the following equations for $F=\psi \gamma_{21} \tilde{\psi}$ :

$$
\partial \psi \gamma_{21} \tilde{\psi}+\left(\widetilde{\partial \gamma_{21}} \tilde{\psi}\right)=2 J_{e}, \quad \partial \psi \gamma_{21} \tilde{\psi}-\left(\widetilde{\partial \gamma_{21}} \tilde{\psi}\right)=2 K_{m},
$$

which is equivalent to (2.12) in [14] (where $\mathscr{G}$ is used for the three form of monopolar current). There, it is observed that $J_{e}$ is even under reversion and $K_{m}$ is odd. Then, it is claimed that "since reversion is a purely algebraic operation without any particular physical meaning, the monopolar current $K_{m}$ is necessarily zero if the Clifford formalism is assumed to provide a representation of Maxwell's equations where the source currents $J_{e}$ and $K_{m}$ correspond to fundamental physical fields." It is also stated that (4.10) and (4.12) impose different constrains on the monopolar currents $J_{e}$ and $K_{m}$.

It is clear that these arguments are fallacious. Indeed, it is obvious that if any comparison is to be made, it must be between $J_{e}$ and $J_{m}$ or between $K_{e}$ and $K_{m}$. In this case, it is obvious that both pairs of currents have the same behavior under reversion. This kind of confusion is widespread in the literature, mainly by people that work with the generalized Maxwell equation(s) in component form (4.9).

It seems that experimentally $J_{m}=0$ and the following question suggests itself: is there any real physical field governed by an equation of the type of the generalized Maxwell equation (1.2)? The answer is yes.

5. The generalized Hertz potential equation. In what follows, we accept that $J_{m}=0$ and take Maxwell equations for the electromagnetic field $F \in$ $\sec \bigwedge^{2}(M) \subset \sec \mathfrak{b} \ell(M, g)$ and a current $J_{e} \in \sec \bigwedge^{1}(M) \subset \sec \mathfrak{b} \ell(M, g)$ as

$$
\partial F=J_{e}
$$

Let $\Pi=(1 / 2) \Pi^{\mu v} \gamma_{\mu} \gamma_{v}=\vec{\Pi}_{e}+\mathbf{i} \vec{\Pi}_{m} \in \sec \bigwedge^{2}(M) \subset \sec \mathscr{b} \ell(M, g)$ be the socalled Hertz potential [34, 37]. We write

$$
\left[\Pi^{\mu \nu}\right]=\left[\begin{array}{cccc}
0 & -\Pi_{e}^{1} & -\Pi_{e}^{2} & -\Pi_{e}^{3} \\
\Pi_{e}^{1} & 0 & -\Pi_{m}^{3} & \Pi_{m}^{2} \\
\Pi_{e}^{2} & \Pi_{m}^{3} & 0 & -\Pi_{m}^{1} \\
\Pi_{e}^{3} & -\Pi_{m}^{2} & \Pi_{m}^{1} & 0
\end{array}\right]
$$

and define the electromagnetic potential by

$$
A=-\delta \Pi \in \sec \Lambda^{1}\left(T^{\star} M\right) \subset \sec \mathscr{b} \ell(M, g) .
$$


Since $\delta^{2}=0$, it is clear that $A$ satisfies the Lorenz gauge condition, that is,

$$
\delta A=0
$$

Also, let

$$
\gamma^{5} S=d \Pi \in \sec \bigwedge^{3}(M) \subset \sec \mathfrak{b} \ell(M, g),
$$

and call $S$ the Stratton potential. It follows also that

$$
d\left(\gamma^{5} S\right)=d^{2} \Pi=0 .
$$

But $d\left(\gamma^{5} S\right)=\gamma^{5} \delta S$, from which we get, taking into account (4.10),

$$
\delta S=0
$$

We can put (5.3) and (5.5) in the form of a single generalized Maxwell-like equation, that is,

$$
\partial \Pi=(d-\delta) \Pi=A+\gamma^{5} S=\mathscr{A} .
$$

Equation (5.8) is the equation we were looking for. It is a legitimate physical equation. We also have

$$
\square \Pi=(d-\delta)^{2} \Pi=d A+\gamma_{5} d S .
$$

Next, we define the electromagnetic field by

$$
F=\partial \mathscr{A}=\square \Pi=d A+\gamma_{5} d S=F_{e}+\gamma_{5} F_{m} .
$$

We observe that

$$
\square \Pi=0 \Longrightarrow F_{e}=-\gamma_{5} F_{m} .
$$

Now, we calculate $\partial F$. We have

$$
\partial F=(d-\delta) F=d^{2} A+d\left(\gamma^{5} d S\right)-\delta(d A)-\delta\left(\gamma^{5} d S\right) .
$$

The first and last terms in the second right member of (5.9) are obviously null. Writing

$$
J_{e}=-\delta d A, \quad \gamma^{5} J_{m}=-d\left(\gamma^{5} d S\right),
$$

we get Maxwell equation

$$
\partial F=(d-\delta) F=J_{e}
$$

if and only if the magnetic current $\gamma^{5} J_{m}=0$, that is,

$$
\delta d S=0
$$


a condition that we suppose to be satisfied in what follows. Then,

$$
\square A=J_{e}=-\delta d A, \quad \square S=0 .
$$

Now, we define

$$
F_{e}=d A=\vec{E}_{e}+\mathbf{i} \vec{B}_{e}, \quad F_{m}=d S=\vec{B}_{m}+\mathbf{i} \vec{E}_{m}
$$

and also

$$
F=F_{e}+\gamma_{5} F_{m}=\vec{E}+\mathbf{i} \vec{B}=\left(\vec{E}_{e}-\vec{E}_{m}\right)+\mathbf{i}\left(\vec{B}_{e}+\vec{B}_{m}\right) .
$$

Then, we get

$$
\square \vec{\Pi}_{e}=\vec{E}, \quad \square \vec{\Pi}_{m}=\vec{B} .
$$

It is important to keep in mind that

$$
\square \Pi=0 \Longrightarrow \vec{E}=0, \quad \vec{B}=0 .
$$

Nevertheless, despite this result, we have the following theorem.

HeRTZ THEOREM. The Hertz potential satisfies

$$
\square \Pi=0 \Longrightarrow \partial F_{e}=0 .
$$

Proof. Immediately from the above equations, we have that

$$
\partial F_{e}=-\partial\left(\gamma_{5} F_{m}\right)=-d\left(\gamma_{5} d S\right)+\delta\left(\gamma_{5} d S\right)=\gamma_{5} d^{2} S-\gamma_{5} \delta d S=0 .
$$

We remark that $(5.21)$ has been called the Hertz theorem in $[32,37]$ and it has been used there and also in $[8,9,10,16,20,31,33]$ in order to find nontrivial superluminal solutions of the free Maxwell equation.

6. Maxwell Dirac equivalence of first kind. We consider a generalized Maxwell equation

$$
\partial F=\mathscr{F}
$$

where $\partial=\gamma^{\mu} \partial_{\mu}$ is the Dirac operator and $\mathscr{F}$ is the electromagnetic current (an electric current $J_{e}$ plus a magnetic monopole current $-\gamma_{5} J_{m}$, where $J_{e}$, $\left.J_{m} \in \sec \bigwedge^{1} M \subset \mathscr{C} \ell(M, g)\right)$. We proved in Section 2 that if $F^{2} \neq 0$, then we can write

$$
F=\psi \gamma_{21} \tilde{\psi}
$$


where $\psi \in \sec \mathscr{b} \ell^{+}(M, g)$ is a representative of a Dirac-Hestenes field. If we use (6.2) in (6.1), we get

$$
\partial\left(\psi \gamma_{21} \tilde{\psi}\right)=\gamma^{\mu} \partial_{\mu}\left(\psi \gamma_{21} \tilde{\psi}\right)=\gamma^{\mu}\left(\partial_{\mu} \psi \gamma_{21} \tilde{\psi}+\psi \gamma_{21} \partial_{\mu} \tilde{\psi}\right)=\mathscr{g},
$$

from where it follows that

$$
2 \gamma^{\mu}\left\langle\partial_{\mu} \psi \gamma_{21} \tilde{\psi}\right\rangle_{2}=\mathscr{F}
$$

Consider the identity

$$
\gamma^{\mu}\left\langle\partial_{\mu} \psi \gamma_{21} \tilde{\psi}\right\rangle_{2}=\partial \psi \gamma_{21} \tilde{\psi}-\gamma^{\mu}\left\langle\partial_{\mu} \psi \gamma_{21} \tilde{\psi}\right\rangle_{0}-\gamma^{\mu}\left\langle\partial_{\mu} \psi \gamma_{21} \tilde{\psi}\right\rangle_{4},
$$

and define, moreover, the vectors

$$
j=\gamma^{\mu}\left\langle\partial_{\mu} \psi \gamma_{21} \tilde{\psi}\right\rangle_{0}, \quad g=\gamma^{\mu}\left\langle\partial_{\mu} \psi \gamma_{5} \gamma_{21} \tilde{\psi}\right\rangle_{0} .
$$

Taking into account (6.3), (6.4), (6.5), and (6.6), we can rewrite (6.3) as

$$
\partial \psi \gamma_{21} \tilde{\psi}=\left[\frac{1}{2} \mathscr{g}+\left(j+\gamma_{5} g\right)\right] .
$$

Equation (6.7) is a spinorial representation of Maxwell equation. In the case where $\psi$ is nonsingular (which corresponds to nonnull electromagnetic fields), we have

$$
\partial \psi \gamma_{21}=\frac{e^{\gamma_{5} \beta}}{\rho}\left[\frac{1}{2} \mathscr{f}+\left(j+\gamma_{5} g\right)\right] \psi .
$$

Equation (6.8) representing Maxwell equation, written in that form, does not appear to have any relationship with the Dirac-Hestenes equation (2.42). However, we will make some algebraic modifications on it in such a way as to put it in a form that suggests a very interesting and intriguing relationship between them, and consequently a possible connection between electromagnetism and quantum mechanics.

Since $\psi$ is supposed to be nonsingular $(F \neq 0)$, we can use the canonical decomposition of $\psi$ and write $\psi=\rho e^{\beta_{\gamma_{5}} / 2} R$, with $\rho, \beta \in \sec \bigwedge^{0} M \subset \sec \mathscr{b} \ell(M, g)$ and $R \in \operatorname{Spin}_{+}(1,3)$, for all $x \in M$. Then

$$
\partial_{\mu} \psi=\frac{1}{2}\left(\partial_{\mu} \ln \rho+\gamma_{5} \partial_{\mu} \beta+\Omega_{\mu}\right) \psi,
$$

where we define the 2-form

$$
\Omega_{\mu}=2\left(\partial_{\mu} R\right) \tilde{R}
$$

Using this expression for $\partial_{\mu} \psi$ into the definitions of the vectors $j$ and $g$ (6.6), we obtain that

$$
\begin{aligned}
& j=\gamma^{\mu}\left(\Omega_{\mu} \cdot S\right) \rho \cos \beta+\gamma_{\mu}\left[\Omega_{\mu} \cdot\left(\gamma_{5} S\right)\right] \rho \sin \beta, \\
& g=\left[\Omega_{\mu} \cdot\left(\gamma_{5} S\right)\right] \rho \cos \beta-\gamma_{\mu}\left(\Omega_{\mu} \cdot S\right) \rho \sin \beta,
\end{aligned}
$$


where we define the spin 2-form $S$ by

$$
S=\frac{1}{2} \psi \gamma_{21} \psi^{-1}=\frac{1}{2} R \gamma_{21} \tilde{R} .
$$

We now define

$$
J=\psi \gamma_{0} \tilde{\psi}=\rho v=\rho R \gamma^{0} R^{-1}
$$

where $v$ is the velocity field of the system. To continue, we define the 2 -form $\Omega=v^{\mu} \Omega_{\mu}$ and the scalars $\Lambda$ and $K$ by

$$
\Lambda=\Omega \cdot S, \quad K=\Omega \cdot\left(\gamma_{5} S\right) .
$$

Using these definition, we have that

$$
\Omega_{\mu} \cdot S=\Lambda v_{\mu}, \quad \Omega_{\mu} \cdot\left(\gamma_{5} S\right)=K v_{\mu},
$$

and the vectors $j$ and $g$ can be written as

$$
\begin{aligned}
& j=\Lambda v \rho \cos \beta+K v \rho \sin \beta=\lambda \rho v, \\
& g=K v \rho \cos \beta-\Lambda v \rho \sin \beta=\kappa \rho v,
\end{aligned}
$$

where we defined

$$
\lambda=\Lambda \cos \beta+K \sin \beta, \quad \kappa=K \cos \beta-\Lambda \sin \beta .
$$

The spinorial representation of Maxwell equation is written now as

$$
\partial \psi \gamma_{21}=\frac{e^{\gamma_{5} \beta}}{2 \rho} \mathscr{\psi} \psi+\lambda \psi \gamma_{0}+\gamma_{5} \kappa \psi \gamma_{0}
$$

Observe that there are $[8,9,10,20,31,32,33,34,37]$ infinite families of nontrivial solutions of Maxwell equations such that $F^{2} \neq 0$ (which correspond to subluminal and superluminal solutions of Maxwell equation). Then, it is licit to consider the case $\mathscr{E}=0$. We have

$$
\partial \psi \gamma_{21}=\lambda \psi \gamma_{0}+\gamma_{5} \kappa \psi \gamma_{0}
$$

which is very similar to the Dirac-Hestenes equation.

In order to go a step further into the relationship between those equations, we remember that the electromagnetic field has six degrees of freedom while a Dirac-Hestenes spinor field has eight degrees of freedom, and that we proved in Section 2 that two of these degrees of freedom are hidden variables. We are free therefore to impose two constraints on $\psi$ if it is to represent an electromagnetic field. We choose these two constraints as

$$
\partial \cdot j=0, \quad \partial \cdot g=0
$$


Using (6.16), these two constraints become

$$
\partial \cdot j=\rho \dot{\lambda}+\lambda \partial \cdot J=0, \quad \partial \cdot g=\rho \dot{\kappa}+k \partial \cdot J=0,
$$

where $J=\rho v, \dot{\lambda}=(v \cdot \partial) \lambda$, and $\dot{k}=(v \cdot \partial) k$. These conditions imply that

$$
\kappa \lambda=\lambda \kappa \quad(\lambda \neq 0)
$$

which gives

$$
\frac{\kappa}{\lambda}=\text { const. }=-\tan \beta_{0}
$$

or, from (6.17),

$$
\frac{K}{\Lambda}=\tan \left(\beta-\beta_{0}\right)
$$

Now we observe that $\beta$ is the angle of the duality rotation from $F$ to $F^{\prime}=$ $e^{\gamma_{5} \beta} F$. If we perform another duality rotation by $\beta_{0}$, we have $F \mapsto e^{\gamma_{5}\left(\beta+\beta_{0}\right)} F$, and, for the Takabayasi angle, $\beta \mapsto \beta+\beta_{0}$. If we work therefore with an electromagnetic field duality rotated by an additional angle $\beta_{0}$, the above relationship becomes

$$
\frac{K}{\Lambda}=\tan \beta
$$

This is, of course, just a way to say that we can choose the constant $\beta_{0}$ in (6.23) to be zero. Now, this expression gives

$$
\begin{aligned}
& \lambda=\Lambda \cos \beta+\Lambda \tan \beta \sin \beta=\frac{\Lambda}{\cos \beta}, \\
& \kappa=\Lambda \tan \beta \cos \beta-\Lambda \sin \beta=0,
\end{aligned}
$$

and the spinorial representation of the Maxwell equation (6.19) becomes

$$
\partial \psi \gamma_{21}-\lambda \psi \gamma_{0}=0
$$

Note that $\lambda$ is such that

$$
\rho \dot{\lambda}=-\lambda \partial \cdot J
$$

The current $J=\psi \gamma_{0} \tilde{\psi}$ is not conserved unless $\lambda$ is constant. If we suppose also that

$$
\partial \cdot J=0
$$

we must have

$$
\lambda=\text { const. }
$$


Now, throughout these calculations, we have assumed that $\hbar=c=1$. We observe that, in (6.27), $\lambda$ has the units of (length) $)^{-1}$, and if we introduce the constants $\hbar$ and $c$, we have to introduce another constant with a unit of mass. If we denote this constant by $m$ such that

$$
\lambda=\frac{m c}{\hbar},
$$

then (6.27) assumes a form which is identical to Dirac-Hestenes equation:

$$
\partial \psi \gamma_{21}-\frac{m c}{\hbar} \psi \gamma_{0}=0
$$

It is true that we did not prove that (6.32) is really Dirac equation since the constant $m$ has to be identified in this case with the electron's mass, and we do not have any good physical argument to make that identification, until now. In resume, (6.32) has been obtained from Maxwell equation by imposing some gauge conditions allowed by the hidden parameters in the solution of (1.1) for $\psi$ in terms of $F$. In view of that, it seems more appropriate instead of using the term mathematical MDE of first kind to talk about a correspondence between those equations under which the two extra degrees of freedom of the Dirac-Hestenes spinor field are treated as hidden variables.

To end this section we observe that it is too early to know if the above results are of some physical value or only a mathematical curiosity.

7. Maxwell-Dirac equivalence of second kind. We now look for a Hertz potential field $\Pi \in \sec \bigwedge^{2}(M)$ satisfying the following (nonlinear) equation:

$$
\partial \Pi=\left(\partial \mathfrak{G}+m \mathfrak{\vartheta} \gamma_{3}+m\left\langle\Pi \gamma_{012}\right\rangle_{1}\right)+\gamma_{5}\left(\partial \mathfrak{P}+m \mathfrak{G} \gamma_{3}-\gamma_{5}\left\langle m \Pi \gamma_{012}\right\rangle_{3}\right),
$$

where $\mathfrak{6}, \mathfrak{P} \in \sec \bigwedge^{0}(M)$ and $m$ is a constant. According to Section 5, the electromagnetic and Stratton potentials are

$$
\begin{aligned}
A & =\partial \mathfrak{G}+m \mathfrak{P} \gamma_{3}+m\left\langle\Pi \gamma_{012}\right\rangle_{1}, \\
\gamma_{5} S & =\gamma_{5}\left(\partial \mathfrak{P}+m \mathfrak{G} \gamma_{3}-\gamma_{5}\left\langle m \Pi \gamma_{012}\right\rangle_{3}\right),
\end{aligned}
$$

and must satisfy the following subsidiary conditions:

$$
\begin{gathered}
\square\left(\partial \mathfrak{G}+m \mathfrak{\wp} \gamma_{3}+m\left\langle\Pi \gamma_{012}\right\rangle_{1}\right)=J_{e}, \\
\square\left(\gamma_{5}\left(\partial \mathfrak{\wp}+m \mathfrak{G} \gamma_{3}-\gamma_{5}\left\langle m \Pi \gamma_{012}\right\rangle_{3}\right)\right)=0, \\
\square \mathfrak{G}+m \boldsymbol{\partial} \cdot\left\langle\Pi \gamma_{012}\right\rangle_{1}=0, \\
\square \mathfrak{P}-m \boldsymbol{\partial} \cdot\left(\gamma_{5}\left\langle\Pi \gamma_{012}\right\rangle_{3}\right)=0 .
\end{gathered}
$$


Now, in the Clifford bundle formalism, as we have already explained above, the following sum is a legitimate operation:

$$
\psi=-\mathfrak{G}+\Pi+\gamma_{5} \mathfrak{P}
$$

and, according to the results of Section 2, defines $\psi$ as a representative of some Dirac-Hestenes spinor field. Now, we can verify that $\psi$ satisfies the equation

$$
\partial \psi \gamma_{21}-m \psi \gamma_{0}=0
$$

which is, as we already know, a representative of the standard Dirac equation (for a free electron) in the Clifford bundle, which is a Dirac-Hestenes equation (2.42), written in an orthonormal coordinate spin frame.

The above developments suggest (consistently with the spirit of the generalized Hertz potential theory developed in Section 5) the following interpretation. The Hertz potential field $\Pi$ generates the real electromagnetic field of the electron. (The question of the physical dimensions of the Dirac-Hestenes and Maxwell fields is discussed in [35].) Moreover, the above developments suggest that the electron is composed of two fundamental currents, one of electric type and the other of magnetic type circulating at the ultra microscopic level, which generate the observed electric charge and magnetic moment of the electron. Then, it may be the case, as speculated by Maris [21], that the electromagnetic field of the electron can be split into two parts, each corresponding to a new kind of subelectron-type particle, the electrino. Of course, the above developments leave open the possibility to generate electrinos of fractional charges. We still study more properties of the above system in another paper.

8. Seiberg-Witten equations. As it is well known, the original Seiberg-Witten (monopole) equations have been written in Euclidean spacetime and for the self-dual part of the field $F$. However, on Minkowski spacetime, of course, there are no self-dual electromagnetic fields. Indeed, (2.11) implies that the unique solution (on Minkowski spacetime) of the equation $\star F=F$ is $F=0$. This is the main reason for the difficulties in interpreting that equations in this case, and, indeed, in [38], an interpretation of that equations was attempted only for the case of Euclidean manifolds. Here we want to derive and give a possible interpretation to those equations based on a reasonable assumption.

Now, the analogous of Seiberg-Witten monopole equations reads in the Clifford bundle formalism and on Minkowski spacetime as

$$
\partial \psi \gamma_{21}-A \psi=0, \quad F=\frac{1}{2} \psi \gamma_{21} \tilde{\psi}, \quad F=d A,
$$

where $\psi \in \sec \mathscr{C} \ell^{+}(M, g)$ is a Dirac-Hestenes spinor field, $A \in \sec \bigwedge^{1}(M) \subset$ $\sec \mathscr{C} \ell(M, g)$ is an electromagnetic vector potential, and $F \in \sec \bigwedge^{2}(M) \subset$ $\sec \mathscr{C} \ell(M, g)$ is an electromagnetic field. 
Our intention in this section is

(a) to use the MDE of the first kind (proved in Section 6) and an additional hypothesis to be discussed below to derive the Seiberg-Witten equations on Minkowski spacetime;

(b) to give a (possible) physical interpretation for those equations.

\subsection{Derivation of Seiberg-Witten equations}

STEP 1. We assume that the electromagnetic field $F$ appearing in the second of the Seiberg-Witten equations satisfies the free Maxwell equation, that is, $\partial F=0$.

STEP 2. We use the MDE of the first kind proved in Section 6 to obtain (6.27).

STEP 3. We introduce the ansatz

$$
A=\lambda \psi \gamma_{0} \psi^{-1} \text {. }
$$

This means that the electromagnetic potential (in our geometrical units) is identified with a multiply of the velocity field defined through (6.13). Under this condition, (6.27) becomes

$$
\partial \psi \gamma_{21}-A \psi=0
$$

which is the first Seiberg-Witten equation!

8.2. A possible interpretation of the Seiberg-Witten equations. It is time to find an interpretation for (8.3). In order to do that, we recall from Section 2.5 that if $\psi_{ \pm}$are Weyl spinor fields (as defined through (2.33)), then $\psi_{ \pm}$satisfy a Weyl equation, that is,

$$
\partial \psi_{ \pm}=0 .
$$

Now consider the equation for $\psi_{+}$coupled with an electromagnetic field $A=g B \in \sec \bigwedge^{1}(M) \subset \sec \mathscr{b} \ell(M, g)$, that is,

$$
\partial \psi_{+} \gamma_{21}+g B \psi_{+}=0 .
$$

This equation is invariant under the gauge transformations

$$
\psi_{+} \longmapsto \psi_{+} e^{g \gamma_{5} \theta}, \quad B \longmapsto B+\partial \theta .
$$

Also, the equation for $\psi_{-}$coupled with an electromagnetic field $g B \in$ $\sec \bigwedge^{1}(M)$ is

$$
\partial \psi_{-} \gamma_{21}+g B \psi_{-}=0,
$$

which is invariant under the gauge transformations

$$
\psi_{-} \longmapsto \psi_{-} e^{g \gamma_{5} \theta} ; \quad B \longmapsto B+\partial \theta,
$$


showing clearly that the fields $\psi_{+}$and $\psi_{-}$carry opposite "charges." Now consider the Dirac-Hestenes spinor fields $\psi^{\dagger}, \psi^{\downarrow}$ given by (2.37) which are eigenvectors of the parity operator and look for solutions of (8.3) such that $\psi=\psi^{\dagger}$. We have

$$
\partial \psi^{\dagger} \gamma_{21}+g B \psi^{\dagger}=0
$$

which separates into two equations:

$$
\partial \psi_{+}^{\dagger} \gamma_{21}+g \gamma_{5} B \psi_{+}^{\dagger}=0, \quad \partial \psi_{-}^{\dagger} \gamma_{21}+g \gamma_{5} B \psi_{-}^{\uparrow}=0 .
$$

These results show that when a Dirac-Hestenes spinor field associated with the first of the Seiberg-Witten equations is in an eigenstate of the parity operator, that spinor field describes a pair of particles with opposite charges. We interpret these particles (following Lochak [16] who suggested that an equation equivalent to (8.10) describes massless monopoles of opposite charges) as being massless monopoles in auto-interaction. Observe that our proposed interaction is also consistent with the third of Seiberg-Witten equations, for $F=d A$ implying a null magnetic current.

It is now well known that Seiberg-Witten equations have nontrivial solutions on Minkowski manifolds (see [23]). From the above results, in particular, taking into account the inversion formula (3.12), it seems to be possible to find a whole family of solutions for the Seiberg-Witten equations, which has been here derived from an MDE of first kind (proved in Section 6) with the additional hypothesis that electromagnetic potential $A$ is parallel to the velocity field $v$ (8.2) of the system described by (6.13). We conclude that a consistent set of Seiberg-Witten equations on Minkowski spacetime must be

$$
\begin{gathered}
\partial \psi \gamma_{21}-A \psi=0, \\
F=\frac{1}{2} \psi \gamma_{21} \tilde{\psi}, \quad F=d A, \\
A=\lambda \psi \gamma_{0} \psi^{-1} .
\end{gathered}
$$

9. Conclusions. In this paper, we exhibited two different kinds of possible MDE. Although many will find the ideas presented above speculative from the physical point of view, we hope that they may become important, at least from a mathematical point of view. Indeed, not so long ago, researching solutions of the free Maxwell equation $(\partial F=0)$ satisfying the constraint $F^{2} \neq 0$ (a necessary condition for derivation of an MDE of the first kind) conduced to the discovery of families of superluminal solutions of Maxwell equations and also of all the main linear relativistic equations of theoretical Physics [16, 34]. The study of the MDE of the second kind reveals an unsuspected interpretation of the Dirac equation, namely, that the electron seems to be a composed system built up from the self interaction of two currents of "electrical" and "magnetic" types. 
Of course it is too early to say if this discovery has any physical significance. We also showed that by using the MDE of the first kind together with a reasonable hypothesis, we can shed light on the meaning of Seiberg-Witten monopole equations on Minkowski spacetime. We hope that the results just found may be an indication that Seiberg-Witten equations (which are a fundamental key in the study of the topology of four manifolds equipped with an Euclidean metric tensor) may play an important role in Physics, whose arena, where phenomena occur, is a Lorentzian manifold.

Acknowledgments. The author is grateful to Doctors V. V. Fernández, A. Gsponer, R. A. Mosna, A. M. Moya, I. R. Porteous, and J. Vaz Jr. for useful discussions. He is also grateful to CNPq for a senior research fellowship (Contract 201560/82-8) and to the Department of Mathematical Sciences, University of Liverpool for a Visiting Professor position and hospitality during the academic year 2001/2002.

\section{REFERENCES}

[1] A. A. Campolattaro, New spinor representation of Maxwell equations. II. Algebraic properties, Internat. J. Theoret. Phys. 19 (1980), no. 2, 127-138.

[2] _ New spinor representation of Maxwell's equations. I. Generalities, Internat. J. Theoret. Phys. 19 (1980), no. 2, 99-126.

[3] __ Generalized Maxwell equations and quantum mechanics. I. Dirac equation for the free electron, Internat. J. Theoret. Phys. 29 (1990), no. 2, 141-155.

[4]___ Generalized Maxwell equations and quantum mechanics. II: Generalized Dirac equation, Internat. J. Theoret. Phys. 29 (1990), no. 5, 477-482.

[5] _ Classical electrodynamics and relativistic quantum mechanics, Adv. Appl. Clifford Algebras 7 (1997), suppl., 167-173.

[6] M. Chown, Double or quit, New Scientist 168 (2000), no. 2260, 24-27.

[7] J. P. Crawford, On the algebra of Dirac bispinor densities: factorization and inversion theorems, J. Math. Phys. 26 (1985), no. 7, 1439-1441.

[8] E. C. de Oliveira and W. A. Rodrigues Jr., Superluminal electromagnetic waves in free space, Ann. Physik 7 (1998), 654-659.

[9] _ Finite energy superluminal solutions of Maxwell equations, Phys. Lett. A 291 (2001), no. 6, 367-370.

[10] E. C. de Oliveira, W. A. Rodrigues Jr., D. S. Thober, and A. L. Xavier Jr., Thoughtful comments on: "Bessel beams and signal propagation", Phys. Lett. A 284 (2001), no. 6, 296-303.

[11] Q. A. G. de Souza and W. A. Rodrigues Jr., The Dirac operator and the structure of Riemann-Cartan-Weyl spaces, Gravitation: The Spacetime Structure (Águas De Lindóia, 1993) (P. S. Letelier and W. A. Rodrigues Jr., eds.), World Scientific Publishing, New Jersey, 1994, pp. 179-212.

[12] V. L. Figueiredo, E. C. de Oliveira, and W. A. Rodrigues Jr., Clifford algebras and the hidden geometrical nature of spinors, Algebras Groups Geom. 7 (1990), no. 2, 153-198.

[13] _ Covariant, algebraic, and operator spinors, Internat. J. Theoret. Phys. 29 (1990), no. 4, 371-395.

[14] A. Gsponer, On the "equivalence" of the Maxwell and Dirac equations, Internat. J. Theoret. Phys. 41 (2002), no. 4, 689-694. 
[15] F. Gürsey, Contribution to the quaternion formalism in special relativity, Rev. Fac. Sci. Univ. Istanbul. Sér. A. 20 (1956), 149-171.

[16] G. Lochak, Wave equation for a magnetic monopole, Internat. J. Theoret. Phys. 24 (1985), no. 10, 1019-1050.

[17] P. Lounesto, Clifford algebras and Hestenes spinors, Found. Phys. 23 (1993), no. 9, 1203-1237.

[18] Clifford algebras, relativity and quantum mechanics, Gravitation: the Spacetime Structure (Águas de Lindóia, 1993) (P. S. Letelier and W. A. Rodrigues Jr., eds.), World Scientific Publishing, New Jersey, 1994, pp. 50-81.

[19] A. Maia Jr., E. Recami, W. A. Rodrigues Jr., and M. A. F. Rosa, Magnetic monopoles without string in the Kähler-Clifford algebra bundle: a geometrical interpretation, J. Math. Phys. 31 (1990), no. 2, 502-505.

[20] J. E. Maiorino and W. A. Rodrigues Jr., What is superluminal wave motion? Sci. and Tech. Mag. 2 (1999), no. 4, 1-179.

[21] H. J. Maris, On the fission of elementary particles and the evidence for fractional electrons in liquid helium, J. Low Temp. Phys. 120 (2000), no. 3-4, 173-204.

[22] R. A. Mosna and W. A. Rodrigues Jr., The bundles of algebraic and Dirac-Hestenes spinor fields, RP 57/02, IMECC-UNICAMP, 2002.

[23] G. L. Naber, Topology, Geometry, and Gauge Fields. Interactions, Applied Mathematical Sciences, vol. 141, Springer-Verlag, New York, 2000.

[24] C. Nash, Topology and physics-a historical essay, preprint, 1997, http://arxiv.org/ abs/hep-th/9709135.

[25] G. Y. Rainich, Electrodynamics in the general relativity theory, Trans. Amer. Math. Soc. 27 (1925), no. 1, 106-136.

[26] W. A. Rodrigues Jr., Algebraic and Dirac-Hestenes spinors and spinor fields, RP 56/02, IMECC-UNICAMP, 2002.

[27] W. A. Rodrigues Jr. and E. C. de Oliveira, Dirac and Maxwell equations in the Clifford and spin-Clifford bundles, Internat. J. Theoret. Phys. 29 (1990), no. $4,397-412$.

[28] W. A. Rodrigues Jr. and Q. A. G. de Souza, The Clifford bundle and the nature of the gravitational field, Found. Phys. 23 (1993), no. 11, 1465-1490.

[29] W. A. Rodrigues Jr., Q. A. G. de Souza, and J. Vaz Jr., Spinor fields and superfields as equivalence classes of exterior algebra fields, Clifford Algebras and Spinor Structures (R. Ablamowicz and P. Lounesto, eds.), Math. Appl., vol. 321, Kluwer Academic Publishers, Dordrecht, 1995, pp. 177-198.

[30] W. A. Rodrigues Jr., Q. A. G. de Souza, J. Vaz Jr., and P. Lounesto, Dirac-Hestenes spinor fields on Riemann-Cartan manifolds, Internat. J. Theoret. Phys. 35 (1996), no. 9, 1849-1900.

[31] W. A. Rodrigues Jr. and J.-Y. Lu, On the existence of undistorted progressive waves (UPWs) of arbitrary speeds $0 \leq v<\infty$ in nature, Found. Phys. 27 (1997), no. 3, 435-508.

[32] W. A. Rodrigues Jr. and J. E. Maiorino, A unified theory for construction of arbitrary speeds $(0 \leq v<\infty)$ solutions of the relativistic wave equations, Random Oper. Stochastic Equations 4 (1996), no. 4, 355-400.

[33] W. A. Rodrigues Jr., D. S. Thober, and A. L. Xavier Jr., Causal explanation for observed superluminal behavior of microwave propagation in free space, Phys. Lett. A 284 (2001), no. 6, 217-224.

[34] W. A. Rodrigues Jr. and J. Vaz Jr., Subluminal and superluminal solutions in vacuum of the Maxwell equations and the massless Dirac equation, Adv. Appl. Clifford Algebras 7 (1997), suppl., 457-466. 
[35] - From electromagnetism to relativistic quantum mechanics, Found. Phys. 28 (1998), no. 5, 789-814.

[36] N. Seiberg and E. Witten, Monopoles, duality and chiral symmetry breaking in $N=2$ supersymmetric QCD, Nuclear Phys. B 431 (1994), no. 3, 484-550.

[37] A. L. Trovon de Carvalho and W. A. Rodrigues Jr., The non sequitur mathematics and physics of the "new electrodynamics" proposed by the AIAS group, Random Oper. Stochastic Equations 9 (2001), no. 2, 161-206.

[38] J. Vaz Jr., Clifford algebras and Witten's monopole equations, Geometry, Topology and Physics (Campinas, 1996) (B. N. Apanasov, S. B. Bradlow, W. A. Rodrigues Jr., and K. K. Uhlenbeck, eds.), de Gruyter, Berlin, 1997, pp. 277300.

[39] J. Vaz Jr. and W. A. Rodrigues Jr., Equivalence of Dirac and Maxwell equations and quantum mechanics, Internat. J. Theoret. Phys. 32 (1993), no. 6, 945-959.

[40] _ Maxwell and Dirac theories as an already unified theory, Adv. Appl. Clifford Algebras 7 (1997), suppl., 369-386.

Waldyr A. Rodrigues Jr.: Department of Applied Mathematics, Institute of Mathematics, Statistics and Scientific Computation (IMECC), University of Campinas (UNICAMP), CP 6065, 13083-970 Campinas SP, Brazil

E-mail address: wa7rod@ime.unicamp.br 


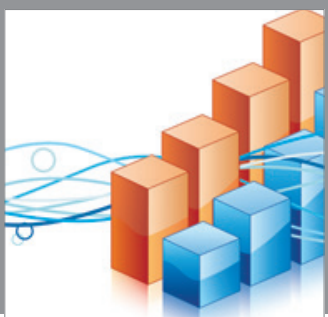

Advances in

Operations Research

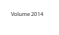

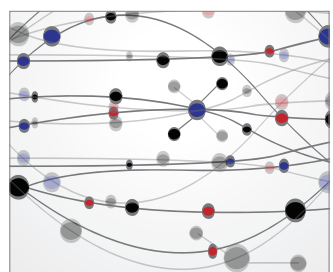

\section{The Scientific} World Journal
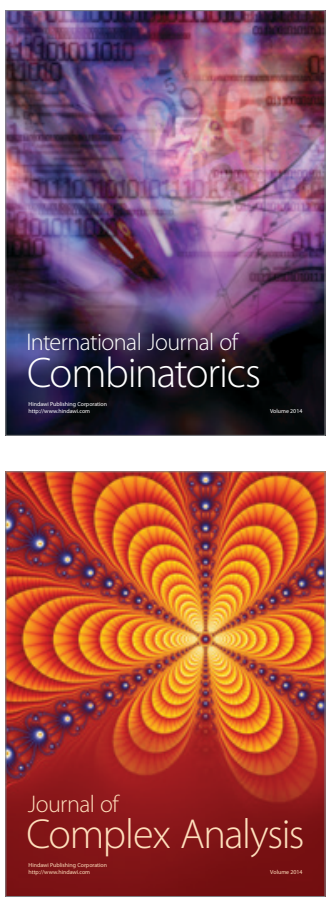

International Journal of

Mathematics and

Mathematical

Sciences
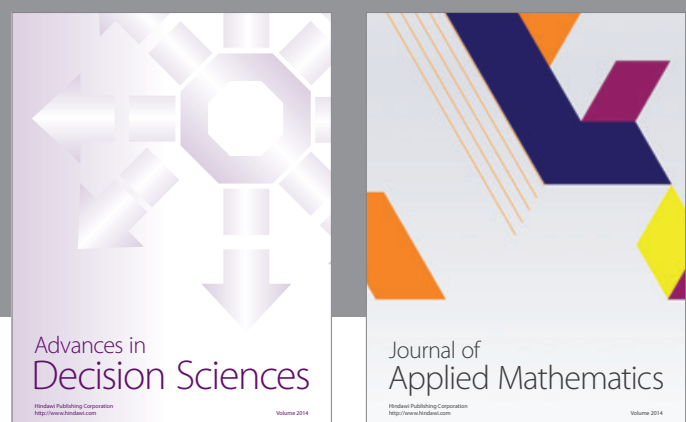

Journal of

Applied Mathematics
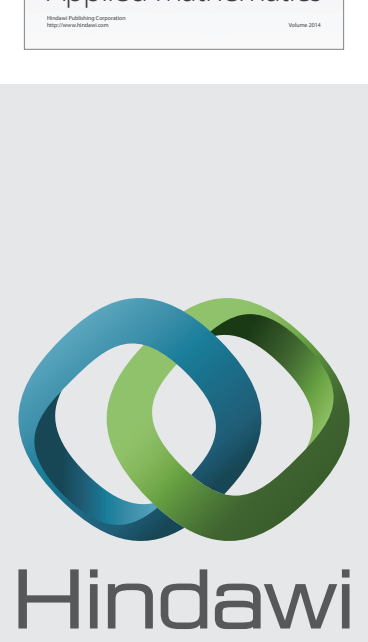

Submit your manuscripts at http://www.hindawi.com
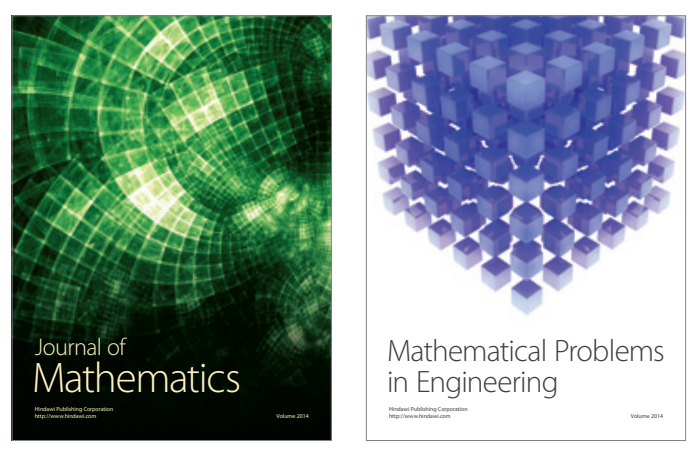

Mathematical Problems in Engineering
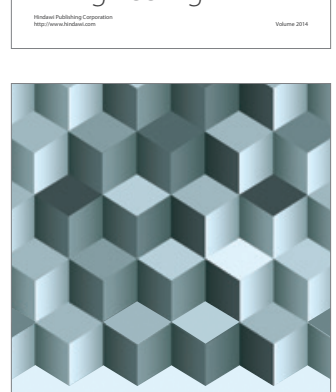

Journal of

Function Spaces
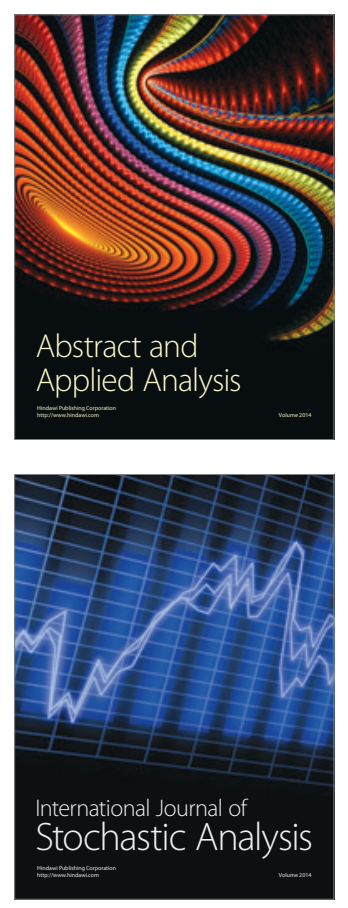

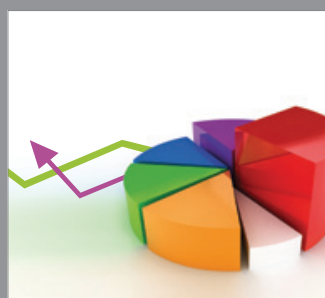

ournal of

Probability and Statistics

Promensencen
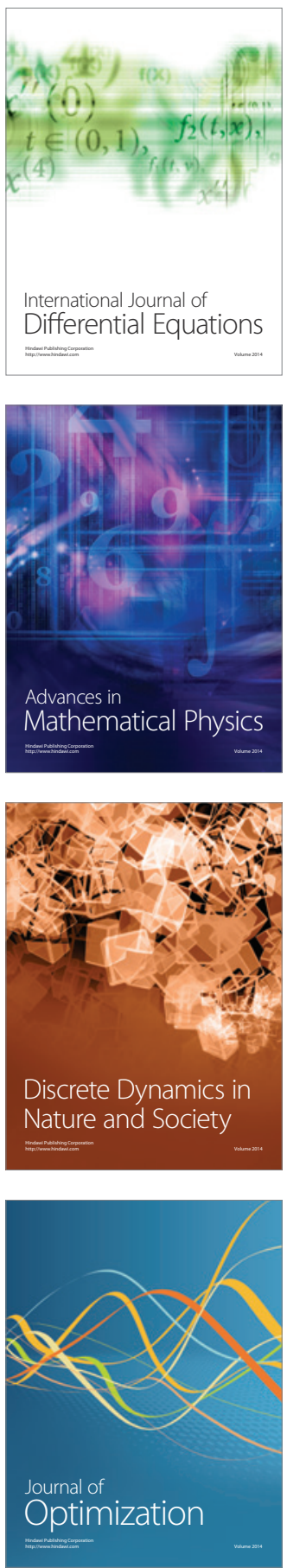